\title{
Enhanced Stochastic Methodology for Combined Architecture of E-Commerce and Security Networks
}

\author{
Song-Kyoo Kim \\ Mobile Communication Division, Samsung Electronics, 94-1 Imsoo-Dong, Gumi, \\ Kyungpook 730-350, South Korea \\ Correspondence should be addressed to Song-Kyoo Kim, amangkim@gmail.com
}

Received 14 April 2009; Accepted 21 June 2009

Recommended by Oleg Gendelman

This paper deals with network architecture which is a combination of electronic commerce and security systems in the typical Internet ecosystems. The e-commerce model that is typically known as online shopping can be considered as a multichannel queueing system. In the other hand, stochastic security system is designed for improving the reliability and availability of the ecommerce system. The security system in this paper deals with a complex system that consists of main unreliable servers, backups, and repair facilities to repair the broken servers. The results are applied for analyzing the current Internet ecosystem, and the mathematical methods can be also applied to the various areas such as human resources, manufacturing processes, and military operations. The solution presents the analytical solutions of the combined architecture of two queueing systems, and these tractable results are used for demonstration in the framework of optimization problems.

Copyright (c) 2009 Song-Kyoo Kim. This is an open access article distributed under the Creative Commons Attribution License, which permits unrestricted use, distribution, and reproduction in any medium, provided the original work is properly cited.

\section{Introduction}

Presently, electronic commerce (e-commerce) has been fastly growing and became extremely popular. Because of heavy traffic on Internet services and Internet shopping, the servers for e-commerce must be available all the time to meet the customer satisfactions. In the case of computer security, there are three distinct concepts which are secrecy (confidentiality), accuracy (integrity), and availability [1]. A secure computer system must not allow information to be disclosed to anyone who is not authorized to access. Accuracy means the system must not corrupt information in the system. Availability means the (computer) system keeps working efficiently and the system is able to recover if a disaster occurs [2]. The availability of network servers is one of the most important issues in the business. The stochastic integrated model is the combination of e-commerce [3] and network security [4] systems under one umbrella (see, Figure 1). Typical electronic commerce service is online 


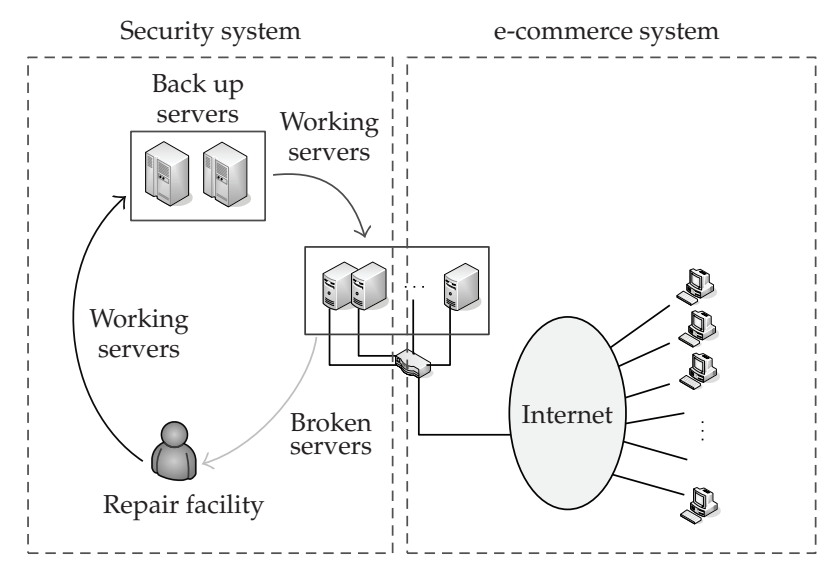

Figure 1: Combined network architecture of two systems.

shopping via Internet [3]. The security system that is also called stochastic backup model [4] is designed for improving the availability and reliability of the network.

As mentioned above, the system that is proposed in the paper consists of two systems. One is a network systems which actually serve customers via Internet and the other is a stochastic security system which supports to maintain main servers using the reserve (spare or backup) servers [2-5].

The e-commerce system has unreliable web-based network servers $(m)$ and each server contains several channels to serve customers. Each customer connects to one of many channels for Internet shopping and a customer leaves when shopping is done. It is assumed that service time of each channel is exponentially distributed with the parameter $\mu$ and identically and independently distributed (iid). The interarrival times of input streams are generally distributed and iid in accordance with the PDF $A(x)$ with the mean $a$ that is the average interarrival time between customer arrivals. Let $Q_{k}(t)$ be the number of occupied channels at time $t$ on the $k$ th server that offers up to $n$ channels. These channels are occupied by customers randomly and iid. The solution of the system can described by a $G I / M / m / 0$ queueing system with embedded chain process and continuous time process. The embedded chain process was developed by Takács [6] and recent researches from Kim [3] deal with the continuous time process of a $G I / M / m / 0$ multichannel open queueing system.

The stochastic security system with reserve backups is designed for improving availability and the reliability by using reserve (or spare backup) servers. A main server is the current server working properly (i.e., open channels, serve customers, etc.). Reserve server (or spares, backups) is a standby server which replaces a main server instantly when it is broken. This system has a repair facility (or called a repairman) to repair the broken server. Independent of any other system, this kind of backup system has been developed with different aspects such as a stochastic reliability system [5], and stochastic disaster recovery system [2].

The paper is organized as follows. Section 2 formalizes the stochastic security system under consideration. Section 3 deals with e-commerce application and shows how to combine the e-commerce and security system. During these two sections, advanced mathematical techniques are applied such as Duality Principle [7], Embedded Markov Process, semiregenerative and supplementary variable techniques to get the solutions of both systems (e-commerce and SSS). Explicit formulas obtained demonstrate a relatively effortless 
use of functionals. These formulas give main stochastic characteristics of the combination system (such as rewards due to customers in e-service system and expenses due to repair, maintenance, waiting, and reward for higher reliability is security system) and optimization of their objective function. Sections 4 and 5 deal with these topics.

\section{Stochastic Security System with Backups}

The stochastic security system can be characterized by the Markovian input with parameter $\lambda$; general-independent service times (i.e., a renewal process) are distributed in accordance with the PDF $B(x)$ and mean $b=\int_{\mathbb{R}} x d B(x)(<\infty)$. The latter is a classical system investigated by Dshalalow [9] and the related tools such as duality principle, Markov chain process, supplementary variable, and semiregenerative techniques are used for analyzing the proposed security system. Let $Z_{t}^{1}$ be the total number of intact main and reserve servers at time $t$. Denote by $Z_{t}$ the total number of main and reserve machines at time $t(\geq 0)$ with a special condition. There are no idle periods even when all main and backup servers become intact.

The system consists of $m$ main server and $w+1$ backup server for security purposes. Denote by $\phi_{k}^{1}:=\lim _{t \rightarrow \infty} P\left\{Z_{t}^{1}=k\right\}$, then the limiting distribution $\phi^{1}=\left(\phi_{0}^{1}, \phi_{1}^{1}, \ldots, \phi_{m+w+1}^{1}\right)$ can be solved as follows:

$$
\begin{aligned}
& \phi_{0}=1-\sum_{n=1}^{m+w} \phi_{n}, \quad k=0, \\
& \phi_{k}=\frac{P_{k-1}}{k a \mu}, \quad k=1, \ldots, m-1, \\
& \phi_{k}=\frac{P_{k-1}}{m a \mu}, \quad k=m, \ldots, m+w,
\end{aligned}
$$

where $\phi_{k}=\lim _{t \rightarrow \infty} P\left\{Z_{t}=k\right\}, k=0,1, \ldots, m+w$. For the process $Z_{t}^{1}$, the corresponding formulas yield

$$
\begin{gathered}
\phi_{k}^{1}=\frac{m \lambda b}{m \lambda b P_{m+w}} \phi_{k}, \quad k=0,1, \ldots, m+w, \\
\phi_{m+w+1}^{1}=\frac{P_{m+w}}{m \lambda b+P_{m+w}} .
\end{gathered}
$$

For the explicit solution, $P_{k}, k=0,1, \ldots, m+w$ can be found as follows:

$$
A^{-1} P_{k}= \begin{cases}\sum_{r=k}^{m-1}(-1)^{r-k}\left(\begin{array}{l}
r \\
k
\end{array}\right) U_{k}, & k=0, \ldots, m-1, \\
Q_{m+w-k}, & k=m, \ldots, m+w,\end{cases}
$$


where

$$
\begin{gathered}
A^{-1}=U_{0}+\sum_{j=0}^{w} Q_{j}, \\
Q(z)=\sum_{i=0}^{\infty} Q_{i} z^{i}=\frac{\beta(m \mu(1-z))(1-z)}{\beta(m \mu(1-z))-z}, \\
\beta(\theta)=\int_{0}^{\infty} e^{-\theta u} B(d u), \\
U_{r}=a_{n} \sum_{r=n+1}^{m} \frac{W_{r}}{a_{r}(1-1 / a \mu r)}, \quad \beta_{r}=\beta(r \mu), \\
b_{s}:=\left\{\begin{array}{l}
\prod_{i=1}^{s} \frac{\beta_{i}}{1-\beta_{i}}, \quad s=1,2, \ldots, \\
1, \quad s=0, \\
S_{j r}=\left(\begin{array}{c}
m \\
r
\end{array}\right)(m b \lambda)^{w+1}\left(\frac{1}{1+m b \lambda}\right)-\sum_{j=0}^{m}(m b \lambda)^{w-j} S_{j r}, \\
r
\end{array}\right)\left[\left(\frac{1}{b \lambda r}\right)\left(\frac{m}{m-r}\right)^{j+1}-\sum_{i=0}^{j+1}\left(\frac{m}{m-r}\right)^{j+1-r}\left(\frac{(m b \lambda)^{r}}{(1+m b \lambda)^{r+1}}\right)\right] .
\end{gathered}
$$

$Q(z)$ of (2.4) is the generating function, convergent in the open disc centered at zero.

\section{Combined Network Architecture of Two Systems}

As the above session mentioned, the e-commerce system is considered as $G I / M / m / 0$ multichannel queueing system but the number of available channels is random. So, a conventional $G I / M / \tilde{m} / 0$ queueing system is proposed rather than $G I / M / m / 0$ system. This system is characterized by general-independent input; $j$ th server has $n$ parallel channels without buffer within unreliable $m$ servers. The channel of each server can be occupied by customers $Q_{j}(t), j=1, \ldots, n$ at time $t$. Since all servers are unreliable, the number of working servers may be less than $m$ and the main servers can be supported by $w+1$ reserve (or backup) servers. Since $Z_{t}^{1}$ is the number of intact servers included with backups, $M_{t}$ gives the number of main intact servers at time $t$. Let $m_{t}$ be total number of channels occupied by customers. It yields

$$
m_{t}=Q_{1}(t)+\cdots+Q_{M_{t}}(t)
$$

where

$$
M_{t}=\min \left\{m, Z_{t}^{1}\right\}
$$


Limiting probabilities $\pi=\left(\pi_{0}, \pi_{1}, \ldots\right)$ (i.e., $\left.\pi_{k}=\lim _{t \rightarrow \infty} P\left\{m_{t}=k\right\}, k=0,1, \ldots\right)$ which are number of occupied channels will be the solution of our system. As we mentioned, service time for each channel is the exponential distribution with parameter $\mu$.

To find the solution, a single-server queueing system which has $n$ available channels is considered. Let $\tau_{0}(=0), \tau_{1}, \ldots$ be interarrival time sequences of interarrival moments of customers and these sequences are iid (identically independently distributed) and the random variable $\sigma_{n}:=\tau_{n+1}-\tau_{n}$ is distributed in accordance with the PDF $A(x)$

$$
\begin{gathered}
A(x):=P\left\{\sigma_{n} \leq x\right\}, \quad x \geq 0, \\
\alpha(\theta):=\mathbb{E}\left[e^{-\theta \sigma_{n}}\right] .
\end{gathered}
$$

The solution of a multichannel queue in a single-server has been developed by Takács [6] and has been applied in the various researches $[2,4,5]$. Limiting probabilities of the $G I / M / m / 0$ multichannel queue $\mathbf{q}=\left(q_{0}, q_{1}, \ldots, q_{n}\right)$ (i.e., $q_{i}:=\lim _{t \rightarrow \infty} P\{Q(t)=i\}, i=0,1, \ldots, n$, and $n$ is the number of channels for each server) are known to satisfy the following formulas (since we assume that the number of channels for servers is iid and all servers are stochastically equivalent, we drop the server index $j$ ):

$$
\begin{gathered}
q_{k}=\frac{1}{k a \mu}\left[\sum_{j=k-1}^{n}\left[\frac{a_{j} B_{n} \sum_{r=j}^{n}\left(\begin{array}{c}
n \\
r
\end{array}\right)}{a_{r}}\right]\left(\begin{array}{c}
j \\
k-1
\end{array}\right)(-1)^{j-k+1}\right], \quad k=1,2, \ldots, n, \\
q_{0}=1-\sum_{l \geq 1} q_{l},
\end{gathered}
$$

where

$$
\begin{aligned}
& B_{n}=\left[\frac{a_{0} \sum_{l=0}^{n}\left(\begin{array}{l}
n \\
l
\end{array}\right)}{a_{l}}\right]^{-1}, \\
& a_{r}:= \begin{cases}1, & r=0, \\
\prod_{i=1}^{r} \frac{1}{1-\alpha_{i}}, & r>0,\end{cases} \\
& \alpha_{r}=\alpha(r \mu), \\
& a=\mathbb{E}\left[\sigma_{n}\right] .
\end{aligned}
$$

Now, we can find the limiting probabilities of this model. The generating function of a singleserver system with multichannels is

$$
\Xi(z)=\lim _{t \rightarrow \infty} \mathbb{E}\left[z^{Q(t)}\right]=\sum_{k=0}^{m} z^{k} q_{k}
$$


and the generating function of number of servers is

$$
\Psi(z)=\lim _{t \rightarrow \infty} \mathbb{E}\left[z^{M_{t}}\right]
$$

From (3.2) and (3.6)-(3.7), we have

$$
\Psi(z)=\lim _{t \rightarrow \infty}\left(\sum_{k=0}^{m} z^{k} P\left\{Z_{t}^{1}=k\right\}+\sum_{k=m+1}^{m+w+1} z^{m} P\left\{Z_{t}^{1}=k\right\}\right)=\sum_{k=0}^{m} z^{k} \phi_{k}^{1}+\sum_{k=m+1}^{m+w+1} z^{m} \phi_{k}^{1} .
$$

To get the solution for a multiserver system with multichannels, we use the generating function as follows:

$$
\Pi(z)=\sum_{k=0}^{(m+w+1) n} z^{k} \pi_{k}=\lim _{t \rightarrow \infty} \mathbb{E}\left[z^{Q_{1}(t)+\cdots+Q_{M_{t}}(t)}\right]
$$

By the conditional expectation, (3.9) yields

$$
\begin{aligned}
\Pi(z) & =\lim _{t \rightarrow \infty} \mathbb{E}\left[\mathbb{E}\left[z^{Q_{1}(t)+\cdots+Q_{M_{t}}(t)} \mid M_{t}\right]\right]=\lim _{t \rightarrow \infty} \mathbb{E}\left[\left(\mathbb{E}\left[z^{Q(t)}\right]\right)^{M_{t}}\right] \\
& =\lim _{t \rightarrow \infty} \mathbb{E}\left[\Xi(z)^{M_{t}}\right]=\Psi(\Xi(z)),
\end{aligned}
$$

and the limiting probabilities are

$$
\pi_{k}=\lim _{z \rightarrow 1} \frac{1}{k !} \frac{d^{k}}{d z^{k}} \Pi(z), \quad k=0,1, \ldots,(m+w+1) n,
$$

which is the limiting distribution $\pi=\left(\pi_{0}, \pi_{1}, \ldots, \pi_{n m+n w+n}\right)$ of the system.

\section{Optimality of the Stochastic Combined System}

In this section we deal with a class of optimization problems that arise in stochastic management. Stochastic optimization method is commonly applied for management problem (see, [2-6]). Let a strategy $\Sigma$ be a set of action we impose on the system (e.g., choice of customer distribution, number of channels in the network server, etc.). For instance, $A(x)$ and $\mu$ can the parts of the function of $m$. On the other hand, a system can be subject to a set, say $C$, of cost functions. Denote by $\Phi(\Sigma, C, t)$ the expected costs within $[0, t]$, due to the strategy $\Sigma$ costs $C$ and define the expected cumulative cost rate over an infinite horizon:

$$
\Phi(\Sigma, C):=\lim _{t \rightarrow \infty} \frac{1}{t} \Phi(\Sigma, C, t) .
$$


We treat this problem as a special case. Since $Q_{k}(t)$ is the number of channels in the $k$ th server and $M_{t}$ is a total number of servers in the system, $m_{t}$ is the number of occupied channels in the systems (from (3.1)) at time $t$. Let $f(n)$ be reward rate from $n$ customers in channels of the servers, which is the same as number of occupied channels. Then the expected reward for all occupied channels in network severs during $[0, t]$ is

$$
U f(t)=\mathbb{E}^{i}\left[\int_{0}^{t} f\left(m_{s}\right) d s\right]=\mathbb{E}^{i}\left[\sum_{j \geq 0} f\left(m_{s}\right) \int_{s=0}^{t} \mathbf{1}_{\{j\}}\left(m_{s}\right) d s\right],
$$

which by Fubini's theorem is

$$
=\sum_{j \geq 0} f(j) \int_{s=0}^{t} P\left\{m_{s}=j\right\} d s
$$

Recall that $Z_{t}^{1}$ is the number of intact servers included in the reserve servers, $M_{t}$ gives the number of the intact working servers at time $t$. Let $g(n)$ be cost rate for $n$ intact main servers. Then the expected cost for all main machines working in the interval $[0, t]$ is

$$
\begin{aligned}
U g(t) & =\mathbb{E}^{i}\left[\int_{0}^{t} g\left(M_{s}\right) d s\right]=\mathbb{E}^{i}\left[\sum_{k=0}^{m+w+1} g(\min (m, k)) \int_{s=0}^{t} \mathbf{1}_{\{k\}}\left(Z_{s}^{1}\right) d s\right] \\
& =\sum_{k=0}^{m+w+1} f_{1}(\min (m, k)) \int_{s=0}^{t} P^{i}\left\{Z_{s}^{1}=k\right\} d s .
\end{aligned}
$$

The number of reserve servers is $W_{t}=Z_{t}^{1}-\min \left\{m, Z_{t}^{1}\right\}$. If $h(n)$ is the cost rate for maintaining $n$ reserve servers, then expected cost for all reserve machines maintained in the interval $[0, t]$ is

$$
U h(t)=\mathbb{E}^{i}\left[\int_{0}^{t} h\left(W_{s}\right) d s\right]
$$

which, after similar calculation, yields

$$
U h(t)=\sum_{k=0}^{m+w+1} h(k-\min (m, k)) \int_{s=0}^{t} P^{i}\left\{Z_{s}^{1}=k\right\} d s
$$


The cumulative cost of all procedures involved in the multichannel system of the network server on the interval $[0, t]$ is

$$
\begin{aligned}
\Phi(\Sigma, C, t)= & U f(t)+U g(t)+U h(t) \\
= & \sum_{j \geq 0} f(j) \int_{s=0}^{t} P\left\{m_{s}=j\right\} d s \\
& -\sum_{k=0}^{m+w+1} g(\min (m, k)) \int_{s=0}^{t} P^{i}\left\{Z_{s}^{1}=k\right\} d s \\
& -\sum_{k=0}^{m+w+1} h(m-\min (m, k)) \int_{s=0}^{t} P^{i}\left\{Z_{s}^{1}=k\right\} d s .
\end{aligned}
$$

Now we turn to convergence theorems for regenerative and semiregenerative processes [9],

(i) $\lim _{t \rightarrow \infty}(1 / t) \int_{0}^{t} P^{i}\left\{m_{s}=k\right\} d s=\pi_{k}$,

(ii) $\lim _{t \rightarrow \infty}(1 / t) \int_{0}^{t} P^{i}\left\{Z_{u}^{1}=k\right\} d u=\phi_{k^{\prime}}^{1}$

to arrive at the objective function $\Phi(\Sigma, C)$, which gives the total expected rate of all processes over an infinite horizon. In light of equations (i)-(ii) we have

$$
\begin{aligned}
\Phi(\Sigma, C) & =\lim _{t \rightarrow \infty} \frac{1}{t} \Phi(\Sigma, C, t) \\
& =\sum_{j \geq 0} f(j) \pi_{j}-\sum_{k=0}^{m+w+1}(g(\min (m, k))+h(m-\min (m, k))) \phi_{k}^{1} .
\end{aligned}
$$

Assuming that all functions are linear, we have

$$
\Phi(\Sigma, C)=d \cdot \widehat{m}-\sum_{k=0}^{m+w+1}(l(\min (m, k))+s \cdot m) \phi_{k^{\prime}}^{1}
$$

where

$$
\widehat{m}=\lim _{t \rightarrow \infty} \mathbb{E}\left[m_{t}\right]=\lim _{t \rightarrow \infty} \mathbb{E}\left[M_{t}\right] \cdot \mathbb{E}[Q(t)]
$$

and $d, l, s$ are relevant (cost and reward) constant coefficients.

\section{Optimization Examples}

In this section we intend to demonstrate the tractability of our results in the previous Sections 2-4 on a practical stochastic integrated model of e-commerce and security system. The exponential distribution is allowed for customer input and broken rate of servers. 
To demonstrate a practical optimization method, we specify the remaining of the three primary cost and reward functions as follows:

$$
f(n)=c_{1} \cdot n, \quad g(n)=c_{2} \cdot n, \quad h(n)=c_{3} \cdot n .
$$

From (5.1) and (4.9), we have

$$
\Phi(\Sigma, C)=c_{1} \cdot \widehat{m}-\sum_{k=0}^{m+w+1}\left(c_{2} \min (k, m)+c_{3}(m-\min (k, m))\right) \phi_{k^{\prime}}^{1}
$$

where $\widehat{m}=\lim _{t \rightarrow \infty} \mathbb{E}\left[m_{t}\right]$. Finally, we arrive at the following expression for the objective function:

$$
\Phi(\Sigma, C)=c_{1} \cdot \widehat{m}-\left(c_{2}-c_{3}\right) \sum_{k=0}^{m}(k-m) \phi_{k}+c_{2} m
$$

Comparing (4.8) with (5.3), we identify $d=c_{1}, l=c_{2}-c_{3}$, and $s=c_{2}$. We restrict the initial strategy of this model to a combination of the number of channels $(n)$ and main $(m)$ and backup $(w)$ servers. In other words, we need to find the triad $\left(n_{0}, m_{0}, w_{0}\right)$ such that

$$
\phi\left(\Sigma\left(n_{0}, m_{0}, w_{0}\right), C\right)=\max \{\phi(\Sigma(n, m, w), C)\}
$$

There is one more constraint under which we set the total number of main and reserve servers as a constant. This constraint is reasonable for limited internal resources for Internet services:

$$
m+w+1=\text { const }
$$

As a reasonable performance measure, let us consider the reliability factor $\rho$, which represents the probability that the number of intact servers at any moment of time in equilibrium is greater than or equal to the number of main servers $(m)$. The reliability factor is defined as follows:

$$
\rho=\sum_{k=m}^{m+w+1} \pi_{k}^{1} .
$$

This is not only a reliability measure of the system, but it can also serve as a constraint to an optimally functioning system.

Figure 2 shows another example of optimization in the system. For graphical illustration, we determine number of backup servers $(w)$ and channels $(n)$. From (5.3), we calculate $\phi(S(m), C)$ and $\left(m_{0}\right)$ that gives a maximum for $\phi(S(m), C)$. Recall that the $m_{0}$ stands for number of main servers which maximizes the total reward of this system when 3 channels are available for each server and $8(w+1)$ backups are available. Below is a plot of $\phi(S(m), C)$ for $m \in\{(m): n=1, \ldots, 10\}$. 


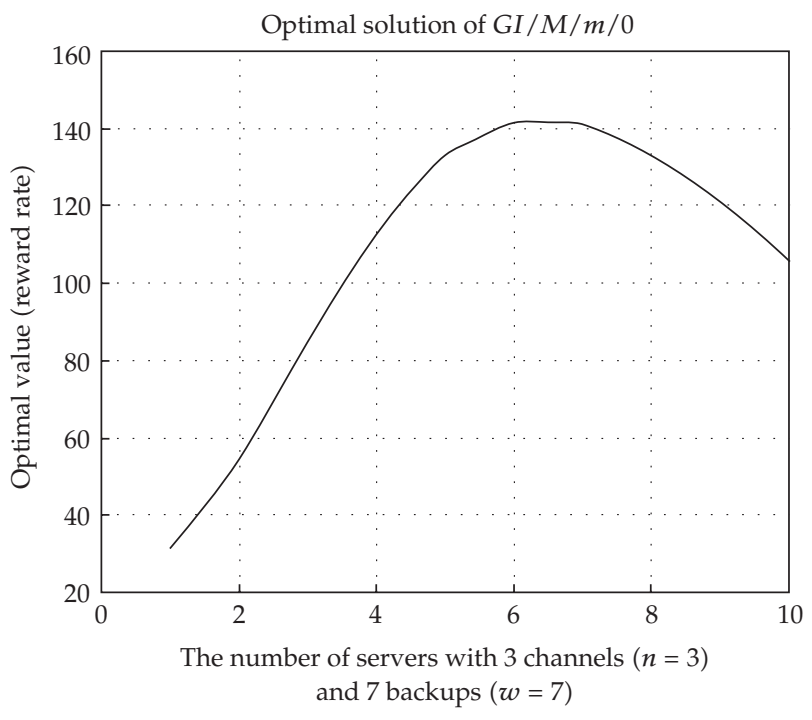

Figure 2: Typical example of stochastic optimization.

The calculation of the objective function yields $m=6$ in maximum reward that equals 141.8249. It means that there are $m_{0}=6$ servers, $8(w+1)$ backups, and 3 available channels in each server, within the system to maximize the reward of maintenance servers.

\section{Conclusions}

The paper provides a method to add a security system to improve availability and reliability issues. The combined architecture considers a combination of two systems. The solution deals with two systems under one integrated system. The objective function of the optimization also contains the properties of two systems.

The paper prepares the full analytic solutions of the integrated model of e-commerce application and security systems which include the embedded Markov process, semi-Markov process, and semiregenerative techniques. These analytic solutions are not only compact but also give all of performance measures of the combination system such as service time and queue length. The optimal number of servers is one example for a demonstration, and the analytic solution can also evaluate a simulation approach of more realistic and complicated system similar with the combined architecture. Applications include not only e-commerce model but also other combinations such as human resources, manufacturing system, and military operations.

\section{References}

[1] D. Russell and G. T. Ganemi Sr., Computer Security Basics, O’Reilly and Asso, Sebastopol, Calif, USA, 1991.

[2] S.-K. Kim and J. H. Dshalalow, "Stochastic disaster recovery systems with external resources," Mathematical and Computer Modelling, vol. 36, no. 11-13, pp. 1235-1257, 2002.

[3] S.-K. Kim, "Stochastic management for randomly broken multi-channel servers for e-commerce applications," in IEEE International Conference on Industrial Engineering and Engineering Management (IEEM 2007 '07), pp. 214-218, Singapore, December 2007. 
[4] S.-K. Kim, "Enhanced networked server management with random remote backups," in Performance and Control of Next-Generation Communications Networks, vol. 5244 of Proceedings of SPIE, pp. 106-114, Orlando, Fla, USA, September 2003.

[5] S.-K. Kim and J. H. Dshalalow, "A versatile stochastic maintenance model with reserve and superreserve machines," Methodology and Computing in Applied Probability, vol. 5, no. 1, pp. 59-84, 2003.

[6] L. Takács, Introduction to the Theory of Queues, University Texts in the Mathematical Sciences, Oxford University Press, New York, NY, USA, 1962.

[7] J. Dshalalow, "On single-server closed queues with priorities and state dependent parameters," Queueing Systems, vol. 8, no. 3, pp. 237-253, 1991.

[8] R. Kalakota, Electric Commerce: A Manager's guide, Addison-Wesley, Reading, Mass, USA, 1997.

[9] J. Dshalalow, "On the multiserver queue with finite waiting room and controlled input," Advances in Applied Probability, vol. 17, no. 2, pp. 408-423, 1985. 


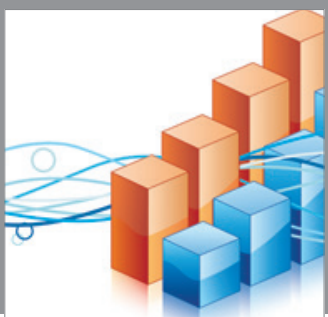

Advances in

Operations Research

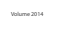

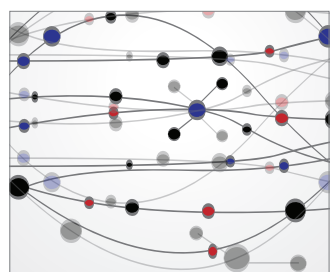

\section{The Scientific} World Journal
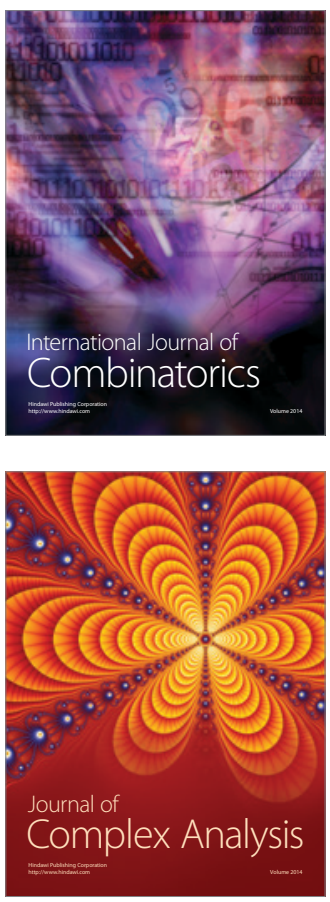

International Journal of

Mathematics and

Mathematical

Sciences
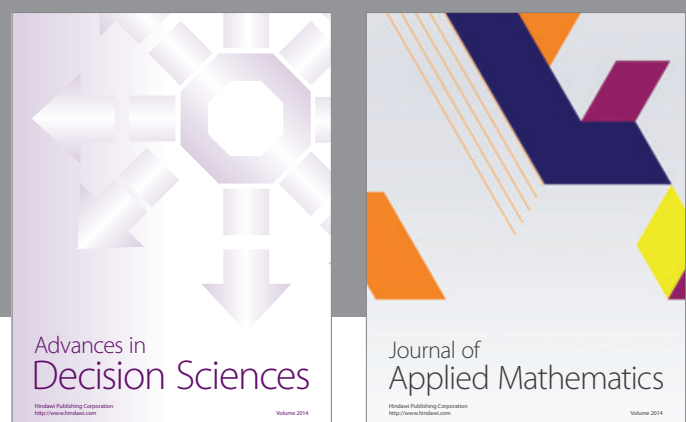

Journal of

Applied Mathematics
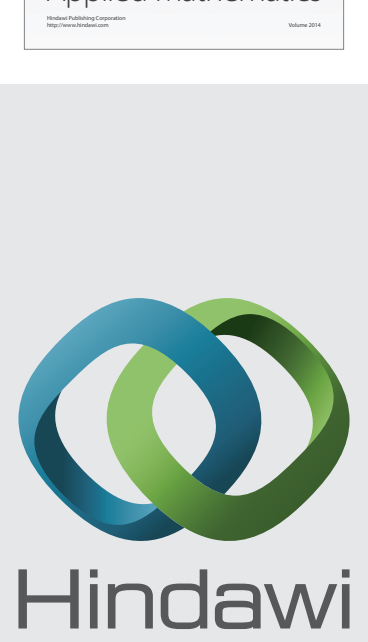

Submit your manuscripts at http://www.hindawi.com
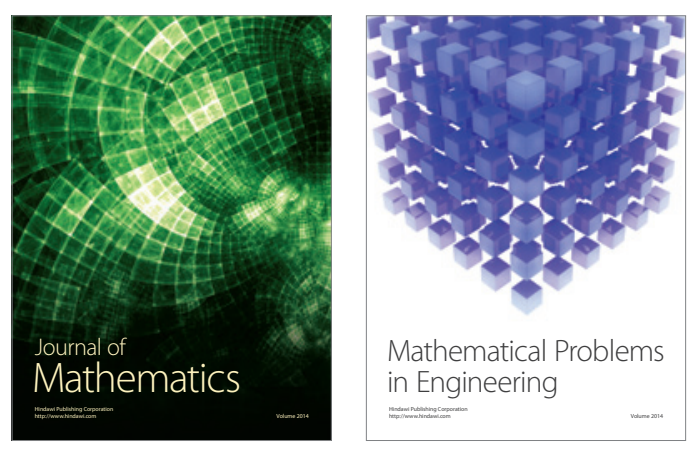

Mathematical Problems in Engineering
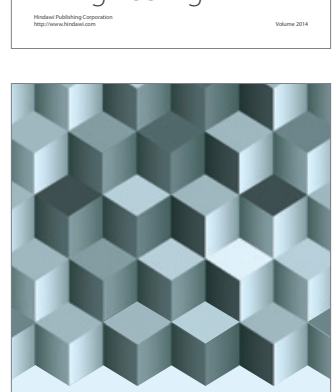

Journal of

Function Spaces
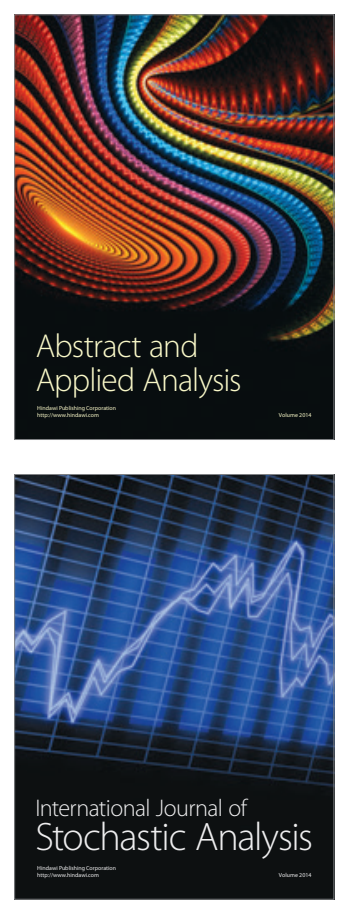

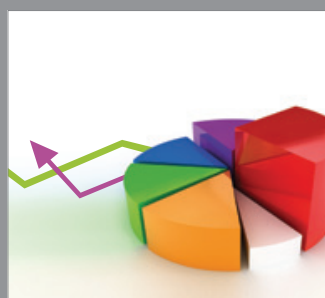

ournal of

Probability and Statistics

Promensencen
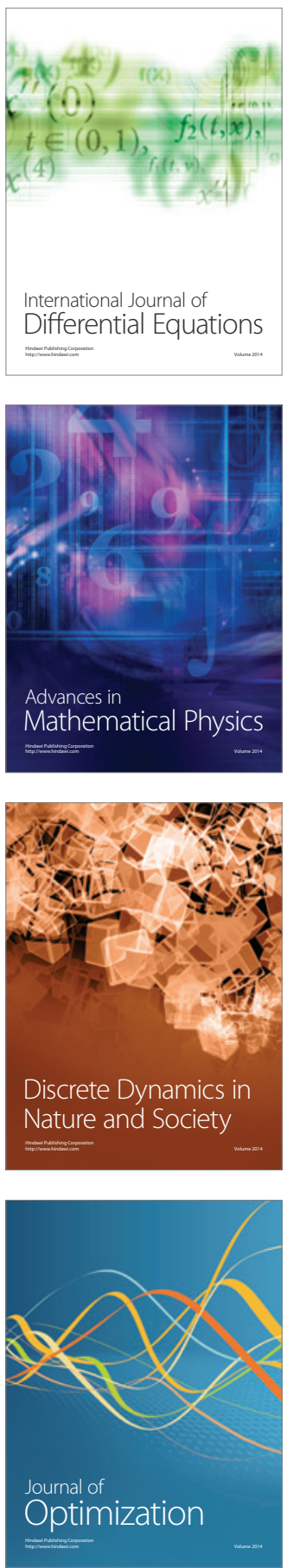